\title{
Research on Network Optimization Based on Simulated Annealing Genetic Algorithm
}

\author{
Xinyun Chen \\ College of Science \&Technology Ningbo University, Ningbo, 315211, \\ China
}

\begin{abstract}
The practicability of the neural network is often affected by the network structure, needing optimization. We introduce the genetic algorithm into the simulated annealing algorithm to propose a new algorithm named the simulated annealing genetic algorithm, which combines the advantages of the two algorithms. The result shows that compared with the genetic algorithm and simulated annealing algorithm, the number of iterations of simulated annealing is fewer and the time of iterations is less.

Keywords:simulated annealing algorithm, genetic algorithm, network optimization
\end{abstract}

\section{Introduction}

The neural network is a kind of active edge sexual intercourse subject which is developed on the basis of many subjects. On one hand, it is influenced by other disciplines, which in turn will affect the development of other disciplines. From the point of view of information theory, neural network is another tool for information processing. On the other hand, the direct application of modern science in the new theory and new methods, at the same time for these disciplines, many new problems, will promote the development of these disciplines and other aspects of the theory. Neural network is not only a highly nonlinear dynamic system, but also an adaptive organization system. Mapping the input pattern to the corresponding classifier is represented by the weights. At first, the weight of the given problem domain is unknown, and the neural network cannot solve the problem until it finds a useful weight. The process of finding useful weights is 
called training. The first is to provide a training set, which consists of the input sample and the corresponding output representing the correct classification. There is a difference between supervised and unsupervised learning for each vector in the training set that has a specific desired output. Network training process, including from the training set to the weights of the mapping. At least in the given error, the weights of the training set can be classified correctly. In fact, the network is trained to teach. If the training set is selected reasonably, and the training algorithm is effective, the network should be able to correctly classify the inputs that do not belong to the training set. From the development and application of the neural network, it can be seen that the neural network theory has a wide range of applications, so the problems of BP neural network are studied deeply. In this paper, the genetic algorithm based on simulated annealing is used to optimize the neural network in order to promote the application of neural network in other fields.

\section{Simulated annealing genetic algorithm}

\subsection{Simulated annealing algorithm}

The basic idea of the simulated annealing algorithm is derived from the principle of annealing in physics, the object will be heated to a high temperature and then let it cool. General solid when the temperature rises inside the atom will become active as temperatures rise and disorder, can also increase, but with the decrease of the temperature of active atoms will decrease and atomic activities become orderly, reaches the equilibrium state within a certain temperature gradually reduced to the minimum. The physical annealing process is used to simulate the optimization problem, and the assumption that the internal energy of the solid is an objective function, and the annealing temperature is assumed to be the control parameter of the algorithm, is the basic simulated annealing algorithm. The simulated annealing algorithm is usually calculated by the initial value and control parameters, and the iterative process of the current solution is carried out. The approximate solution is obtained when the iteration is stopped. The simulated annealing algorithm includes three functions of two criteria, namely state producing function, acceptance function, temperature update function, inner loop and outer loop termination criteria for termination criteria, these factors will optimize the performance of simulated annealing algorithm. In addition, the choice of initial temperature has a great influence on the performance of the simulated annealing algorithm. The simulated annealing algorithm to optimize the effect of a decisive factor is the initial temperature according to the actual value, to optimize the calculation problem, feasible in a certain temperature range, the initial temperature is much higher probability of getting the global optimal solution is greater, but the high initial temperature value will also bring a huge amount of computation need to calculate the time spent will be more. In the actual operation, the initial temperature of the control is not good, need to be adjusted for many times to get satisfactory results. 


\subsection{Genetic algorithm}

The genetic algorithm is a computational model of biological evolution process of natural selection and genetic mechanism of the simulation of Darwin's theory of biological evolution, is a kind of method to search the optimal solution through simulating the process of natural evolution. A genetic algorithm starts with a population that represents a possible set of solutions to a problem, while a population is composed of a number of individuals that are encoded by a gene. Each individual is actually a chromosome with a characteristic entity. Chromosome is the main carrier of genetic material, which is a collection of multiple genes. It determines the external manifestations of the shape of an individual. Therefore, it is necessary to realize the mapping from phenotype to genotype in the beginning. Because of the complexity of modeling the genes, we tend to simplify them, such as binary coding. After the initial population has, in accordance with the principle of survival of the fittest and the survival of the fittest, generational evolution produces a better approximate solution. In each generation, individuals are selected according to the fitness of individuals in the problem domain, and the crossover and mutation are performed by means of genetic operators of natural genetics. This process will lead to a group of the same generation of natural evolution population is more than the previous generation to adapt to the environment, the population of the best individual after decoding, can be used as the approximate optimal solution problem. The genetic algorithm uses the chromosome in biology to encode the optimized object into a data chain to form a chromosome". Encoding and decoding are the main components of genetic algorithms. According to the rule of natural selection, the genetic algorithm is used to eliminate the optimal solution is obtained.

\subsection{Summary of simulated annealing genetic algorithm}

The global search ability of genetic algorithm is easy to fall into the local optimal solution, and the simulated annealing algorithm is outstanding in the global search. The advantages of combining two kinds of optimization algorithm of genetic algorithm and simulated annealing algorithm form optimization algorithm of complementary advantages. The optimization calculation efficiency will be greatly improved. The combination of genetic algorithm and simulated annealing algorithm, genetic algorithm can effectively solve the practical difficulties in the selection and implementation strategy; acceptance probability on individual chromosomes produced, not only improves the ability of global search optimization algorithm to the optimal solution and optimization algorithm in the calculation of the late strong climbing ability, faster convergence the speed optimization of the latter; the introduction of the traditional genetic algorithm group thought in the simulated annealing algorithm. The algorithm can perform multiple local search in the solution space, not only to accelerate the searching speed of the algorithm and improve the optimization ability in dealing with the problem of local convergence of the simulated annealing algorithm; genetic algorithm is used to control optimization the direction, speed up the search 
process; using the simulated annealing algorithm to handle the problem of local convergence and improve the search accuracy. It makes full use of the local search ability of simulated annealing algorithm and the fast global search ability of genetic algorithm.

\section{Network optimization experiment by simulated annealing genetic algorithm}

\subsection{Problem description}

We use the parallel simulated annealing genetic algorithm, design a network and train it to recognize 26 English letters. The network error range is 0.01 Boolean grids and each letter corresponds to a $5 \times 7$. The input vector has 35 elements; the number of samples is 26; the output vector is 26 elements. Each an element corresponds to a letter.

\subsection{Optimization steps}

We use the Matlab software to implement the simulated annealing genetic algorithm. The steps of the simulated annealing genetic algorithm are as follows:

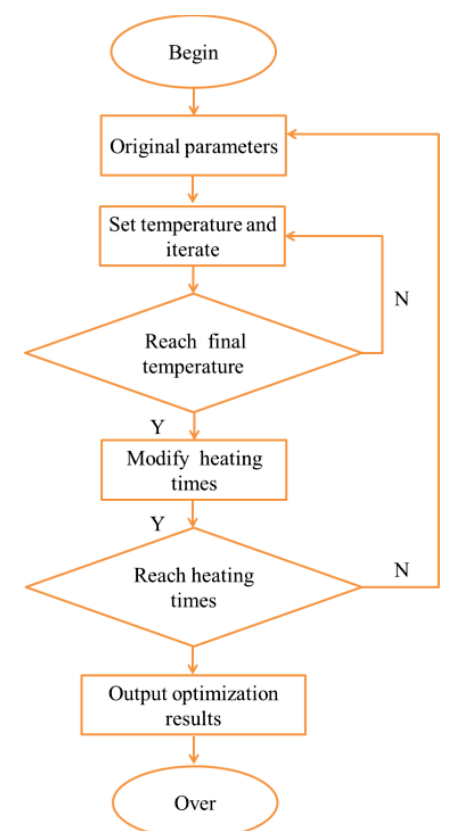

Fig. 1: Steps of simulated annealing genetic algorithm 


\subsection{Simulation results}

We use Matlab programming to achieve the above improved simulated annealing genetic algorithm. We use simulated annealing algorithm, genetic algorithm and simulated annealing genetic algorithm to train the neural network, and apply the trained network to 26 English letters. We use the number of iterations to converge to the global optimal solution as a measure of the convergence rate of the algorithm. The simulated annealing algorithm study, after 3610 times of training to achieve; to get the weights and thresholds of classical genetic algorithm with simulated annealing algorithm, learning the same, after 2126 iterations, the overall network error can reach the expected value; and by the proposed algorithm to obtain the weights and threshold, learning algorithm using the same, after 781 iterations to converge to the optimal solution. The comparison of the three algorithms is shown in Table 1.

Table 1: Comparison of three algorithms

\begin{tabular}{|c|c|c|}
\hline & $\begin{array}{c}\text { Number of } \\
\text { iterations }\end{array}$ & $\begin{array}{c}\text { Time of } \\
\text { iterations }\end{array}$ \\
\hline Simulated Annealing Algorithm & 3610 & 798 \\
\hline Genetic Algorithm & 2126 & 401 \\
\hline $\begin{array}{c}\text { Simulated Annealing Genetic } \\
\text { Algorithm }\end{array}$ & 781 & 62 \\
\hline
\end{tabular}

\section{Conclusion}

The algorithm proposed in this paper is better than the simulated annealing algorithm and the classical genetic algorithm. The optimization speed is faster than the above two algorithms. The improvement of neural network optimization is a continuous process, which needs further study.

\section{References}

[1]Shao Xing, Wang Ruchuan, Huang Haiping, Sun Lijuan, Research of Network Coding Optimization Based onSimulated Annealing Genetic Algorithm, Journal of Nanjing University of Posts and Telecommunications(Natural Science), 33(2), pp. 80-85, 2013.

[2] Gu Liangling, Wang Yuhan, Image enhancement based on simulated annealing algorithm and genetic algorithm, Laser Journal, 36(2), pp. 19-22, 2015. [3]He Guohua, Xie Jiancang, Wang Ni, Zhu Jiwei, Yang Liu, Wang Deyang, Optimal allocation of water resources based on simulatedannealing-genetic algorithm, Journal of Northwest A \& F University(Natural Science Edition), 44(6), pp. 196-202, 2016. 
[4] Lu Xi, Mo Tingting, Parallel Test Task Scheduling Based onSimulated Annealing Tabu Search Genetic Algorithm, Microelectronics \& Computer, 32(3), pp. 146-150, 2015. 